\title{
Can forward dynamics simulation with simple model estimate complex phenomena?: Case study on sprinting using running-specific prosthesis
}

\author{
Akihiko Murai ${ }^{*} \mathbb{D}$, Hiroaki Hobara, Satoru Hashizume, Yoshiyuki Kobayashi and Mitsunori Tada
}

\begin{abstract}
Surpassing the world record in athletic performance requires extensive use of kinematic and dynamic motion analyses to develop novel body usage skills and training methods. Performance beyond the current world record has not been realized or measured; therefore, we need to generate it with dynamics consistency using forward dynamics simulation, although it is technologically difficult because of the complexity of the human structure and its dynamics. This research develops a multilayered kinodynamics simulation that uses a detailed digital human model and a simple motion-representation model to generate the detailed sprinting performances of individuals with lower extremity amputations (ILEAs) aided by carbon-fiber running-specific prostheses (RSPs), which have complex interactions with humans. First, we developed a digital human model of an ILEA using an RSP. We analyzed ILEA sprinting based on experimental motion measurements and kinematics/dynamics computations. We modeled the RSP-aided ILEA sprinting using a simple spring-loaded inverted pendulum model, comprising a linear massless spring, damper, and mass, and we identified the relevant parameters from experimentally measured motion data. Finally, we modified the sprint motion by varying the parameters corresponding to the RSP characteristics. Here, the forward dynamics have been utilized to simulate detailed whole-body sprinting with different RSP types (including simulated RSPs not worn by the subject). Our simulations show good correspondence with the experimentally measured data and further indicate that the sprint time can be improved by reducing the RSP viscosity and increasing stiffness. These simulation results are validated by the experimentally measured motion modifications obtained with different types of RSPs. These results show that the multilayered kinodynamics simulation using the detailed digital human model and the simple motion-representation model has the capacity to generate complex phenomena such as RSP-aided ILEA sprinting that contains complex interactions between the human and the RSP. This simulation technique can be applied to RSP design optimization for ILEA sprinting.
\end{abstract}

Keywords: Digital human technology, Running-specific prosthesis, Motion modification simulation

\section{Introduction}

We have measured the kinetic and physiological aspects of human performance using an optical motion capture system, force plate, etc., and applied kinematics and dynamics analyses to compute the joint angles and

\footnotetext{
*Correspondence: a.murai@aist.go.jp

Digital Human Research Group, National Institute of Advanced Industrial Science and Technology (AIST), 2-3-26, Aomi, Koto-ku, Tokyo 135-0064, Japan
}

(c) The Author(s) 2018. This article is distributed under the terms of the Creative Commons Attribution 4.0 International License (http://creativecommons.org/licenses/by/4.0/), which permits unrestricted use, distribution, and reproduction in any medium, provided you give appropriate credit to the original author(s) and the source, provide a link to the Creative Commons license, and indicate if changes were made. torques and estimate muscle activities, in the fields of biomechanics and sports science. This method has realized excellent athletic performances and clarified injury mechanisms, although it cannot analyze the performances that have not been realized, for instance, a performance that surpasses the current world record. We usually generate the motions of robots, for instance, a grounded manipulator with dynamic consistency, by (1) joint angle or task-based motion generation and (2) forward dynamics simulation. However, applying this

\section{Springer Open}


technique to human whole-body motion generation is considerably difficult, because (1) humans have many more degrees of freedom and a much more complicated structure compared to robots, and (2) humans are floating systems; therefore, we need to estimate the contact forces that can easily become unstable, especially during dynamic motions such as sprinting [1-4]. This research solves these problems by developing a multilayered kinodynamics simulation that uses a detailed digital human model and a simple motion-representation model, which parametrically represents human motion mechanisms. Here, kinodynamics represents the discipline that tries to solve kinematic constraints and dynamic constraints simultaneously, as defined in [5]. In this study, we analyzed and modelled the sprinting performances of individuals with lower extremity amputations (ILEAs), aided by carbon-fiber running-specific prostheses (RSPs), which entail complex interactions between humans and RSPs that form the kinematic and dynamic constraints, to improve the RSP-aided ILEA sprinting performance.

Carbon-fiber RSPs have enabled ILEAs to realize hitherto unachieved degrees of high-level sprinting [6]. While the running mechanics in able-bodied sprinters and ILEAs have been previously examined, these researches were mainly limited to biomechanical studies [7, 8]. Further, many studies have investigated the RSP behavior and performance during sprinting through rigid-body dynamics [9] and finite element analysis [10], but the relationship between the RSP characteristics and sprint performance remains unclear. In particular, RSP-aided ILEA sprinting involves humans and RSPs, as well as the kinematic and dynamic interactions between them, and its kinematics and dynamics analyses are technically complex compared with those of general rigid body systems that are often analyzed in the robotics field. We generated the RSP-aided ILEA sprinting motion, which contains the complex interactions between humans and RSPs, by developing a multilayered kinodynamics simulation, which uses a detailed digital human model and a simple motion-representation model that parametrically represents human motion mechanisms.

Digital human models have been developed to study body kinematics and perform dynamics analyses. These models have been developed based on the knowledge of human anatomy, and they can estimate and analyze human motion through kinematics and dynamics computations [11-14]. We extended the spring-loaded inverted pendulum (SLIP) model for the simple motionrepresentation model that parametrically represents human motion mechanisms. The sprinting motion is often simplified using the SLIP model, which models the entire human body as a spring-mass model and describes the spring-like leg movement during sprinting [15]. We

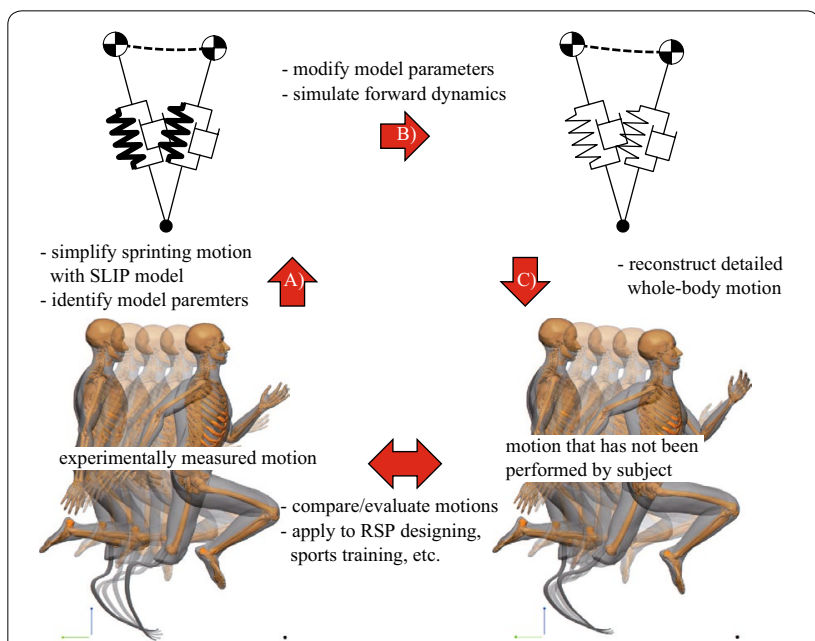

Fig. 1 Concept chart of multilayered kinodynamics simulation of motion modification. This simulation consists of three steps: A simplify the sprinting motion with the SLIP model and identify its parameters using experimentally measured motion, B modify the identified model parameters and simulate the forward dynamics of the simple model, and $\mathrm{C}$ reconstruct the detailed whole-body motion from the motion of the simple model

applied a unilateral SLIP model with a spring, damper, and mass, similar to the one used in [16], to model the RSP-aided ILEA sprinting motion. Figure 1 shows the concept of multilayered kinodynamics simulation. This simulation consists of (A) simplification of sprint motion by using the SLIP model comprising a spring, damper, and mass, and identification of the relevant parameters from experimentally measured motion, (B) modification of the sprint motion by varying the SLIP model parameters and simulation of its forward dynamics, and (C) reconstruction of the detailed whole-body sprint motion from the simulated SLIP model motion. Our approach realizes the simulation of the detailed whole-body sprinting of the specific subject using different RSP types and properties (including simulated RSPs not worn by the subject). We evaluated our simulation results by comparing them with the experimentally measured motion, and both result sets showed good correspondence. This modeling and simulation technique can contribute to the quantitative evaluation and design of RSPs to realize higher levels of RSP-aided 'ILEA sprinting' performances.

\section{Methods}

We first modified our anatomographic digital human model [11] by adjusting the surface shape and skeleton, so that the model represented the kinematic and dynamic characteristics of the ILEAs using the RSPs. The ablebodied individual and ILEA models consisted of 18 and 23 bones, respectively. Each bone was represented as 
a rigid-body linkage with inertial parameters, and the bones were connected to each other via spherical joints. Figure 2 shows the digital human model of an able-bodied individual and an ILEA with unilateral transfemoral amputation wearing an RSP (Sprinter 1E90, Ottobock, Duderstadt, Germany, and Xtreme, Ossur, Reykjavik, Iceland) with a prosthetic knee joint (3S80, hydraulic singleaxis knee joint, Ottobock, Duderstadt, Germany) (male, height $1.68 \mathrm{E}+00 \mathrm{~m}$ and weight $7.01 \mathrm{E}+01 \mathrm{~kg}$ for an ablebodied individual and $6.65 \mathrm{E}+01 \mathrm{~kg}$ for the ILEA).

Next, ILEA sprinting with the unilateral transfemoral amputation was captured by means of a commercial marker-based optical motion capture system with 20 cameras (VICON, Oxford, England) operating at a frame rate of $200 \mathrm{~Hz}$. The subject wore two types of RSPs (Sprinter 1E90 and Xtreme) with the prosthetic knee joint (3S80). The subject was free from any injuries at the time of data collection, and our study protocol was approved by the local institutional review board and conformed to the guidelines outlined in the Declaration of Helsinki (1983). The subject wore 57 markers, whose locations were determined based on an improved version of the Helen Hayes Hospital marker set. Further, 20 additional markers were attached to each RSP to capture the detailed RSP deformation. The positions of the markers are indicated in Fig. 2 by means of white spheres. We also recorded the contact force between the ILEA and the floor using seven force plates (AMTI, MA, USA), each of which measured the six-axis contact force and momentum at a rate of $2 \mathrm{kHz}$. The inverse kinematics was computed with DhaibaWorks [17], and the inverse dynamics was solved with OpenSim [12]. The multilayered kinodynamics simulation for motion modification consisted of the following three steps (Fig. 1).

\section{(A) Simplification of sprint motion using the SLIP model and identification of relevant parameters}

The sprinting was analyzed with simplified models to extract the kinematic and dynamic characteristics to realize stable dynamic simulations. The SLIP model, which represents the entire human body as a springmass model, has been previously applied to describe the spring-like leg movement during locomotion and sprinting [15]. In this study, we applied the unilateral 'springdamper-mass SLIP model' to represent RSP-aided ILEA sprinting (Fig. 3). Here, the whole body was modeled as a mass supported by a spring and damper connected in parallel. Next, we identified the relevant parameters of this model using experimentally measured motion data. The natural length of the leg $\left(L_{\text {leg }, 0}\right)$ and the spring and damper parameters ( $K_{\text {leg }}$ and $D_{\text {leg }}$, respectively) were identified for the intact limb and the RSP, respectively, by mathematical optimization. This optimization minimized the error between the spring and damper forces and measured the contact force between the ground and the intact limb or the RSP, as given in the following equation:

$$
\begin{aligned}
E_{f}= & \sum_{t}^{T}\left(F_{\text {leg }}(t)-\left(K_{\text {leg }}\left(L_{\text {leg }}(t)-L_{\text {leg }, 0}\right)\right.\right. \\
& \left.\left.+D_{\text {leg }} L_{\text {leg }}(t)\right)\right)^{2}
\end{aligned}
$$

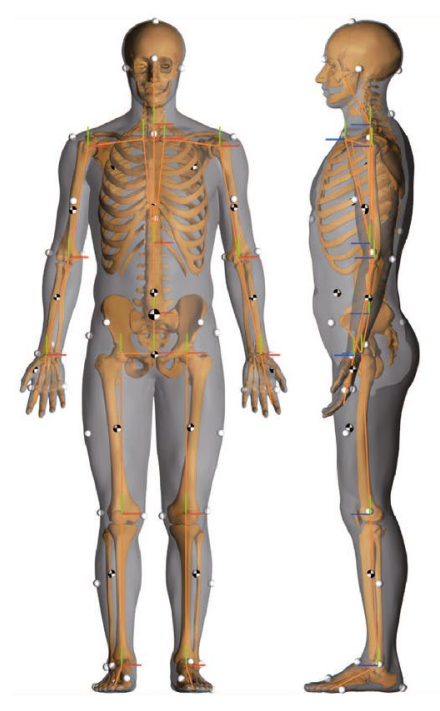

able-bodied model

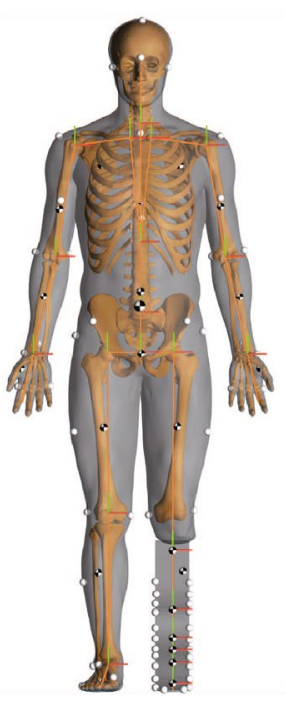

RSP (Sprinter 1E90)

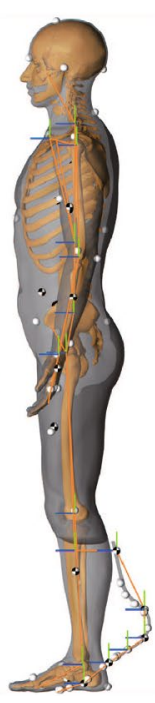

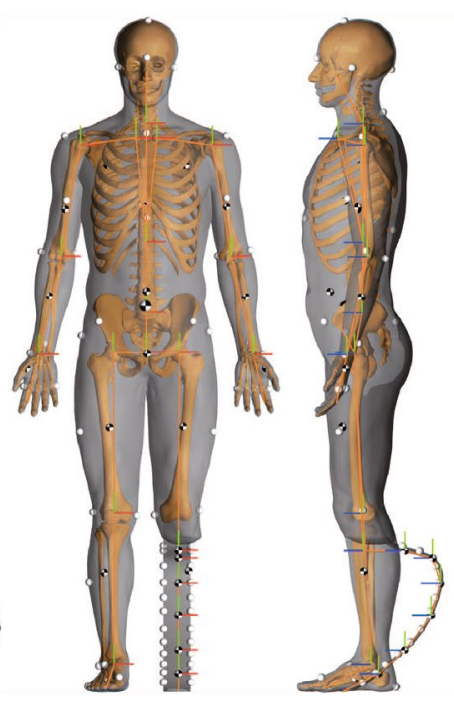

RSP (Xtreme)

Fig. 2 Digital human models of an able-bodied individual and ILEA fitted with an RSP. Left: digital human model of the able-bodied individual, middle: model of the ILEA fitted with Sprinter 1E90, and right: model of the ILEA fitted with Xtreme 


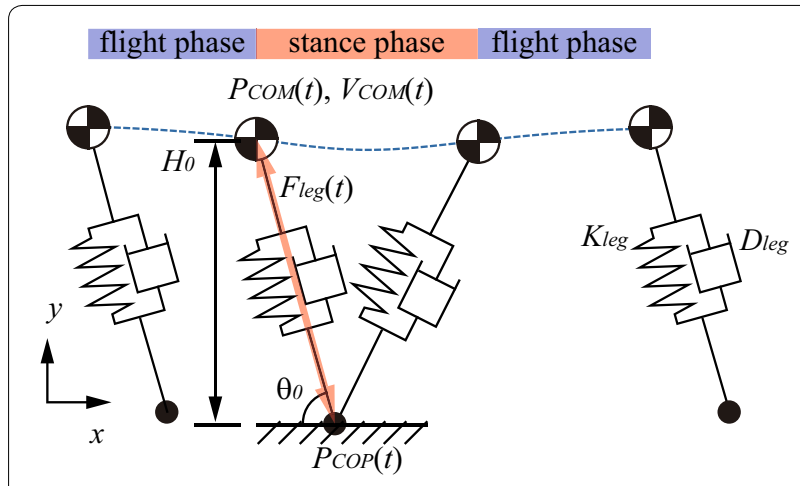

Fig. 3 SLIP model for RSP-aided 'ILEA sprinting'

Table 1 Parameters for the RSPs and intact leg in the SLIP model

\begin{tabular}{llllll}
\hline & Prosthetic & & & \multicolumn{1}{l}{ Intact } \\
\cline { 2 - 3 } & Sprinter 1E90 & Xtreme & & Sprinter 1E90 & Xtreme \\
\hline$L_{\text {leg, } 0}(\mathrm{~m})$ & $1.05 \mathrm{E}+00$ & $1.06 \mathrm{E}+00$ & & $1.06 \mathrm{E}+00$ & $1.06 \mathrm{E}+00$ \\
$K_{\text {leg }}(\mathrm{N} / \mathrm{m})$ & $1.69 \mathrm{E}+04$ & $2.04 \mathrm{E}+04$ & & $2.14 \mathrm{E}+04$ & $2.25 \mathrm{E}+04$ \\
$D_{\text {leg }}(\mathrm{Ns} / \mathrm{m})$ & $8.77 \mathrm{E}+00$ & $9.23 \mathrm{E}+01$ & & $2.92 \mathrm{E}+01$ & $2.01 \mathrm{E}+01$ \\
$H_{0}(\mathrm{~m})$ & $9.41 \mathrm{E}-01$ & $9.81 \mathrm{E}-01$ & & $9.63 \mathrm{E}-01$ & $9.96 \mathrm{E}-01$ \\
$\theta_{0}(\mathrm{rad})$ & $1.34 \mathrm{E}+00$ & $1.31 \mathrm{E}+00$ & & $1.37 \mathrm{E}+00$ & $1.36 \mathrm{E}+00$ \\
\hline
\end{tabular}

where $F_{\text {leg }}$ is the measured contact force, $L_{\text {leg }}$ is the measured length of the leg, and $L_{\text {leg }}$ is its velocity. We also computed the height $\left(H_{0}\right)$ of the center of mass $(\mathrm{COM})$ and the contact angle $\left(\theta_{0}\right)$ at forefoot strike for each case from the experimental data.

\section{(B) Modification and simulation of the forward dynamics of RSP-aided ILEA sprinting}

The kinematic and dynamic characteristics of an RSP can be modified by changing its shape and material. We varied the SLIP model parameters that correspond to the RSP characteristics, $\left(K_{\text {leg }}\right.$ and $\left.D_{\text {leg }}\right)$. Here, we remark that RSP-aided ILEA sprinting is the result of complex interactions between the human controller and the RSP characteristics. The identified parameters in "Results and discussion" (Table 1) indicate that all the parameters, except the RSP characteristics ( $K_{\text {leg }}$ and $D_{\text {leg }}$ of the prosthesis), yield similar values during sprinting with different RSPs. Therefore, we assumed that this specific subject utilizes the same control strategy for all RSPs, and only the parameters corresponding to the RSP characteristics change when the individual wears a different type of RSP. We simulated ILEA sprinting using different types of RSPs by modifying $K_{l e g}$ and $D_{l e g}$ of the prosthesis and computed the forward dynamics. Here, we computed COM acceleration $\left(A_{C O M}(t)\right)$ in the following process.

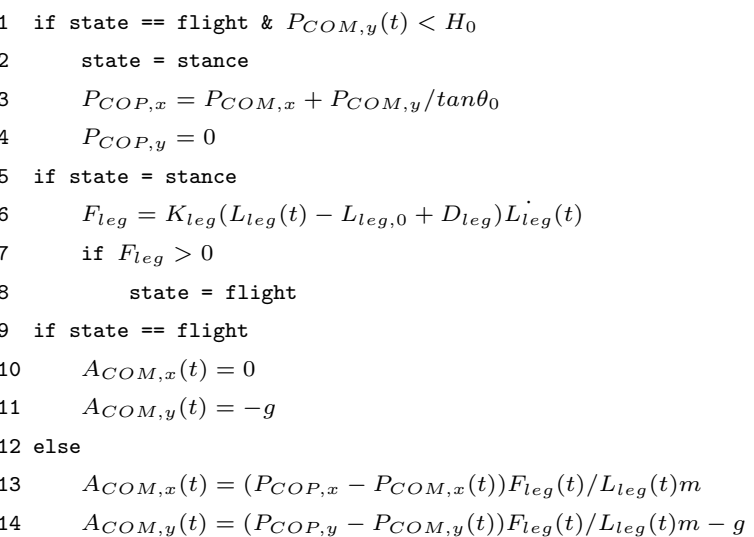

where state represents the phase of sprinting and $m$ represents the total mass of the body. $K_{\text {leg }}$ and $D_{\text {leg }}$ change depending on whether the intact or the prosthetic leg is in contact with the ground. The time integration of $A_{C O M}(t)$ computes the trajectory of COM and COP in this forward dynamics simulation.

\section{(C) Reconstruction of detailed whole-body motion from the simulated SLIP model motion}

We reconstructed the detailed whole-body motion from the simulated simple SLIP model motion for detailed kinematics and dynamics analyses and visualization. The trajectories of all 77 markers, which were experimentally measured, were represented using the quadratic form of the SLIP model status. The parameters of this mapping function from the SLIP model status to the trajectories of all 77 markers were optimized by minimizing the following function:

$$
\begin{aligned}
E_{m}= & \sum_{t}^{T}\left(P_{m a r}(i, j, t)\right. \\
& \left.-M\left(P_{C O M, j}(t), P_{C O P, j}(t)\right)\right)^{2},
\end{aligned}
$$

where $P_{\text {mar }}$ is the measured marker position, $i$ is the marker ID, $j \in(x, y, z)$, and $M$ is the quadratic form of the COM position $\left(P_{C O M}\right)$ and the position of the center of pressure $(\mathrm{COP})\left(P_{\mathrm{COP}}\right)$, as given in the following function:

$$
\begin{aligned}
& M\left(P_{C O M, j}(t), P_{C O P, j}(t)\right) \\
& =\left(\alpha\left(P_{C O M, j}(t)-P_{C O P, j}(t)\right)+\beta\right)^{2} .
\end{aligned}
$$

We optimized the parameters $\alpha$ and $\beta$ to minimize the evaluation function $E_{m}$. The trajectories of the 77 markers were reconstructed from the SLIP model motion in the forward dynamics simulation, the abovementioned parameters, and the kinematics constraints arising from the COP position using this $M\left(P_{C O M, j}, P_{C O P, j}\right)$. This step 
significantly contributes to the detailed kinematics and dynamics analyses. The whole-body joint angles were estimated using the inverse kinematics computation performed using these marker trajectories and the detailed digital human model. The whole-body joint torques were estimated using the inverse dynamics computation performed using these joint angles and the contact forces that were estimated in step (B) using the SLIP model.

\section{Results and discussion}

We can observe the following points from the experimental results:

1. Figure 4 shows the analyzed motion of RSP-aided ILEA sprinting (Sprinter 1E90). Our model simulates the COM trajectories with an average error of $1.94 \mathrm{E}+01 \mathrm{~mm}$ during the stance phase of sprinting. The kinematics and dynamics of the digital human model compute both the human joint torque and bending torque of the RSP during sprinting using its shape and the external force acting upon it. Figure 5 illustrates the bending moment at each point of the RSPs. With regard to step (A) in "Introduction", Table 1 lists the parameters identified from the stance phases of the Sprinter 1E90 and Xtreme RSPs and the intact leg. The values of the prosthetic parameters $K_{\text {leg }}$ and $D_{\text {leg }}$ exhibit apparent differences, which correspond to the RSP characteristics, although the other parameters yield similar values.

2. Our model identifies these parameters with average errors of $1.04 \mathrm{E}+01 \pm 6.50 \mathrm{E}+00 \%$ and $1.83 \mathrm{E}+01 \pm$ $1.40 \mathrm{E}+01 \%$ (average $\pm \mathrm{SD}$ ) in the contact forces for Sprinter $1 \mathrm{E} 90$ and Xtreme, respectively. In step (B), the COM trajectories during the RSP-aided ILEA sprinting (Sprinter 1E90) was simulated (Fig. 4). Our model simulated the COM trajectories with error of $1.94 \mathrm{E}+01 \pm 5.08 \mathrm{E}+00 \mathrm{~mm}$ (average $\pm \mathrm{SD}$ ) during the stance phase of sprinting. In step (C), we reconstructed the whole-body 77-marker positions through steps (A to $\mathrm{C}$ ) without changing the

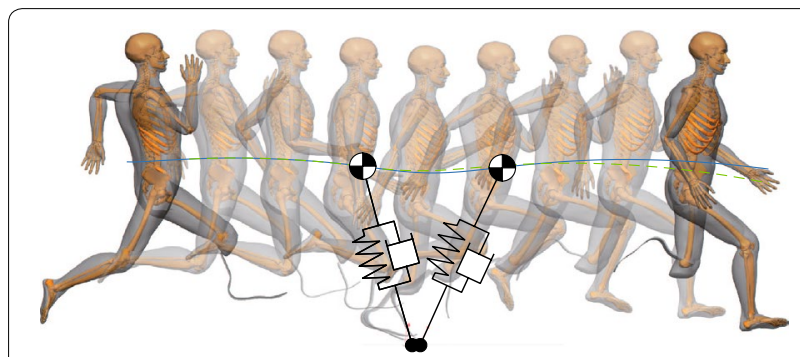

Fig. 4 Synthesized motion of ILEA sprinting using RSP (20 fps). Blue: measured center of mass (COM) trajectory; green: simulated COM trajectory

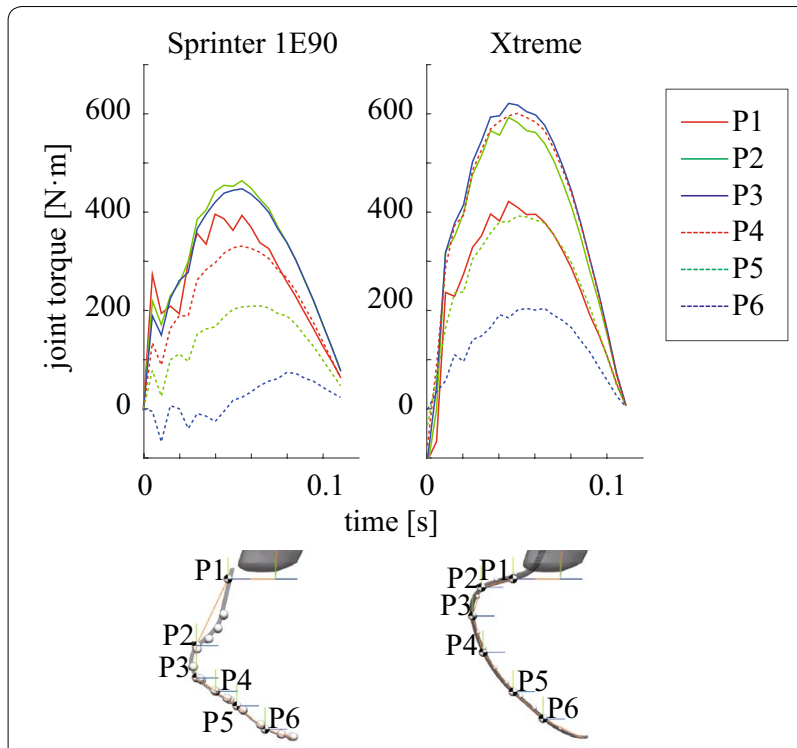

Fig. 5 RSP torques during sprinting. Horizontal axis: time, the origin of which represents the instant of the left forefoot strike, and vertical axis: flexion/extension torque whose positive value represents flexion. Left: Sprinter $1 \mathrm{E} 90$ and right: Xtreme. Each line corresponds to a point shown in the corresponding figure at the bottom

SLIP model parameters. Our method reconstructed the marker positions with an error of $4.32 \mathrm{E}+00 \pm$ $1.76 \mathrm{E}+00 \mathrm{~mm}$ (average $\pm \mathrm{SD}$ ), whose maximum errors ranged from $2.20 \mathrm{E}+00 \mathrm{~mm}$ on the marker of the right tragus to $3.02 \mathrm{E}+01 \mathrm{~mm}$ on the marker of the top of the RSP during the stance phase of sprinting.

3. Figure 6 shows the detailed whole-body motion and COM trajectories of ILEA sprinting with different RSP types and properties. Figure 7 shows the hip joint torques at the sides of the RSP during the stance phase of ILEA sprinting with different RSP types and properties, which are the results of the kinematics and dynamics analyses of the detailed wholebody motion. Figure 8 shows the 100-m sprint time obtained with different types of RSPs.

These results have three implications:

1. The appropriate digital human model, motion measurements, and kinematics and dynamics computations aid in realizing dynamics analysis. Figure 5 represents the bending moment at each point of the RSPs during ILEA sprinting. The radii of these curvatures fit well with the values listed in [18]. The SLIP model with the spring, damper, and mass suitably represent the kinematics and dynamic characteristics 

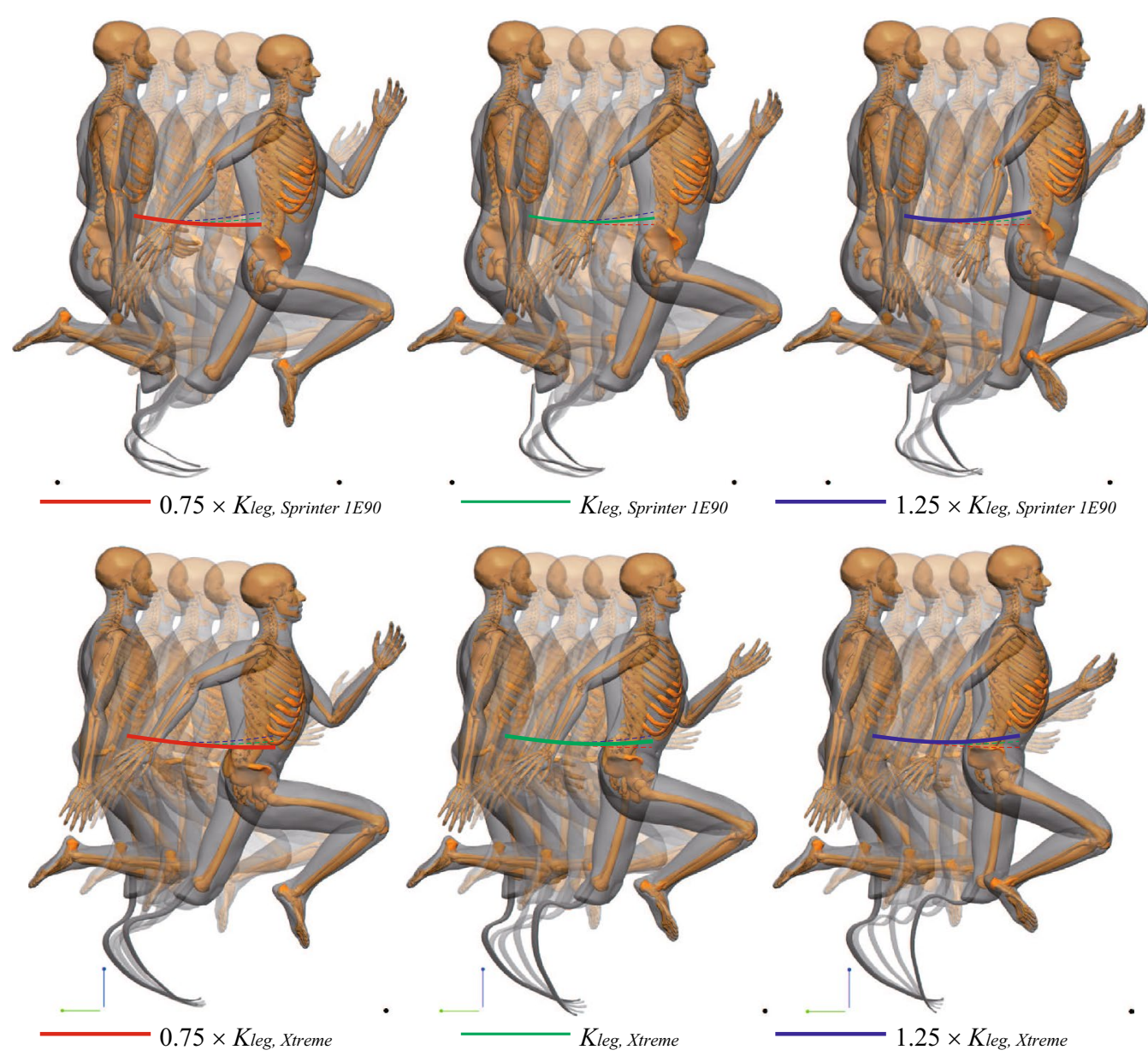

Fig. 6 Simulated whole-body sprint motion and COM trajectory during the stance phase with different RSPs. Top row: Sprinter 1E90, bottom row: Xtreme, left (red): $75 \%$ of $K_{\text {leg, }}$ middle (green): $100 \%$ of $K_{\text {leg, }}$ and right (blue): $125 \%$ of $K_{\text {leg }}$

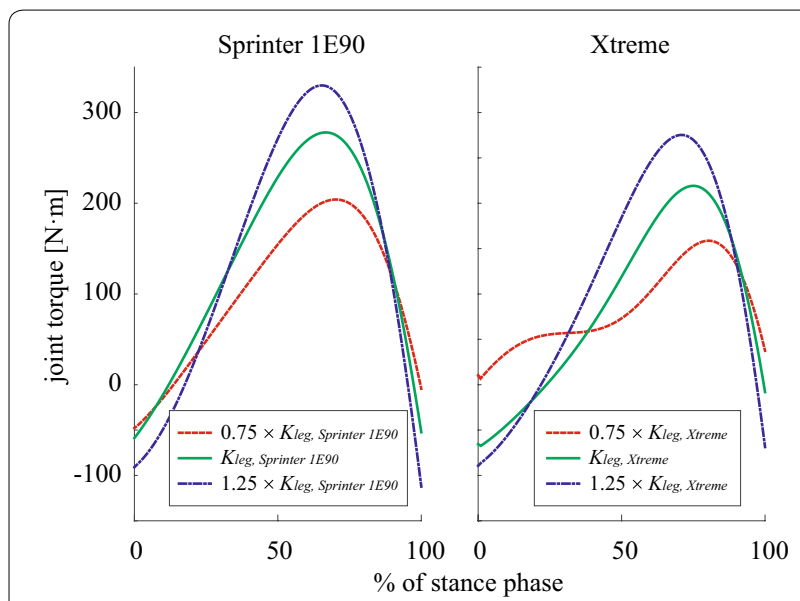

Fig. 7 Simulated left hip joint torque during the stance phase with different types of RSPs. Left graph: Sprinter 1E90, right graph: Xtreme, red dotted line: $75 \%$ of $K_{\text {leg, }}$ green solid line: $100 \%$ of $K_{\text {leg, }}$ and blue dashed-dotted line: $125 \%$ of $K_{\text {leg }}$ of the experimentally measured ILEA sprinting using an RSP for both the intact limb and the RSP.

2. The forward dynamics simulation with the simple SLIP model realizes the kinematics and dynamics analyses of the motions that were not performed by the subject. Figure 6 shows the COM trajectories of ILEA sprinting with different properties of the RSPs. The RSPs with stiffness values of 75 and $125 \%$ were not worn by the subject during the measurements; they were simulated. The simulation results show that both types of RSPs exhibit similar patterns: the subject moves upward when $K_{\text {leg }}$ increases and downward when $K_{l e g}$ decreases. Figure 7 shows the hip joint torques at the sides of the RSPs during the stance phase of the RSP-aided ILEA sprinting with different RSP types and properties, which were the result of the detailed whole-body kinematics and dynamics analyses. The RSPs with stiffness values of 75 and $125 \%$ were not worn by the subject during the 


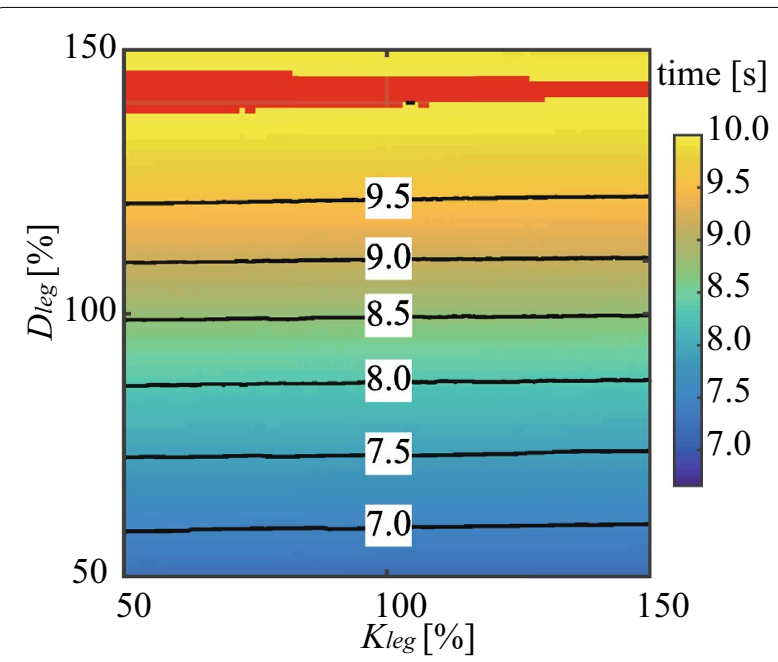

Fig. 8 Time taken for 100-m sprint for the SLIP model with varying $K_{\text {leg }}$ and $D_{\text {leg }}$ values. Horizontal axis: $K_{\text {leg, }}$ vertical axis: $D_{\text {leg }}$ of Sprinter 1E90. The 100-m sprint time with the RSP having the corresponding parameters is represented in a color scale ranging from blue to yellow. The color red indicates that the model falls down before crossing $100 \mathrm{~m}$

measurements; they were simulated. The simulation results show that both types of RSPs exhibit similar patterns: the required hip joint torque increases when the RSP stiffness $\left(K_{\text {leg }}\right)$ increases. Here, we note that the relationship between the RSP stiffness and the required hip joint torque is not a simple linear relationship. The complex relationships in the temporal and amplitude directions appear because of the kinematic and dynamic interactions between humans and the RSPs. The multilayered kinodynamics simulation using the detailed digital human model and simple motion-representation model represents these complex interactions and realizes the non-linear complex relationship between the RSP stiffness and the hip joint torque that is necessary for sprinting using the same control strategy.

3. From Fig. 8, we note that the 100 -m sprint time is significantly improved with decrease in $D_{l e g}$, and the model falls down $\left(P_{C O M, y}(t)\right.$ becomes 0$)$ when $D_{\text {leg }}$ increases drastically. An increase in $K_{\text {leg }}$ also contributes to slightly reducing the sprint time. The sprint time was $8.53 \mathrm{E}+00 \mathrm{~s}$ when $K_{\text {leg }}=1.69 \mathrm{E}+04$ $\mathrm{N} / \mathrm{m}, \quad D_{\text {leg }}=8.77 \mathrm{E}+00 \mathrm{Ns} / \mathrm{m}$ (Sprinter 1E90), and $9.33 \mathrm{E}+00 \mathrm{~s}(9.38 \mathrm{E}+00 \%$ slower $)$ when $K_{\text {leg }}=$ $2.04 \mathrm{E}+04 \mathrm{~N} / \mathrm{m}, D_{\text {leg }}=9.23 \mathrm{E}+01 \mathrm{Ns} / \mathrm{m}$ (Xtreme). The experimentally measured sprint speeds were $7.11 \mathrm{E}+00 \mathrm{~m} / \mathrm{s}$ and $6.60 \mathrm{E}+00 \mathrm{~m} / \mathrm{s} \quad(7.73 \mathrm{E}+00 \%$ slower) for Sprinter 1E90 and Xtreme, respectively.
Here, we first note that the simulated 100-m sprint times were relatively short because of the limitations in our model. One limitation was that a fatigue model was not considered in these simulations. In addition, there were certain dynamic and physical limitations; for instance, the friction parameter and maximum muscle tension have not yet been implemented in our SLIP model. Regardless of the above limitations, our results indicate that the forward dynamics simulation with the simple SLIP model agrees satisfactorily with the measured data at the point that the ratio between the simulated $100-\mathrm{m}$ sprint times using RSPs whose $K_{\text {leg }}$ and $D_{\text {leg }}$ correspond to Sprinter $1 \mathrm{E} 90$ and Xtreme is close to the ratio between the measured sprint speeds using the corresponding RSPs. The simulation results indicate that an increase in $K_{\text {leg }}$ improves the sprint time; therefore, this principle can be applied to RSP design to improve the ILEA sprinting performance. These results, however, are limited to one ILEA sprinter with unilateral transfemoral amputation and using several types of RSPs. Further, we have also assumed that the subject adopts the same control strategy when using different types of RSPs.

\section{Conclusion}

In conclusion, our multilayered kinodynamics simulation realized stable forward dynamics simulation of ILEA sprinting with an RSP on a specific subject, and estimated the detailed kinematic and dynamic characteristics of this complex phenomena. We believe that our approach can contribute to simulating performances that surpass human performances, and particularly contribute to the optimization of RSP design for ILEA sprinting.

\section{Authors' contributions}

In this study, AM performed the model development, kinematics/dynamics computations, and data analysis, and participated in acquiring the measurements. $\mathrm{HH}, \mathrm{SH}$, and YK performed the measurements and helped in drafting the manuscript. MT performed the software development. All authors read and approved the final manuscript.

\section{Acknowledgements}

This research was supported by a Grant-in-Aid for Young Scientists (A) \#17H04700 and Scientific Research(A) \#939778.

\section{Competing interests}

The authors declare that they have no competing interests.

Ethics approval and consent to participate

Written informed consent was obtained from the patient for the publication of this report and any accompanying images.

\section{Funding}

This research was supported by a Grant-in-Aid for Young Scientists (A) \#17H04700 and Scientific Research(A) \#939778. 


\section{Publisher's Note}

Springer Nature remains neutral with regard to jurisdictional claims in published maps and institutional affiliations.

Received: 31 January 2018 Accepted: 8 May 2018

Published online: 18 May 2018

\section{References}

1. Taylor GW, Hinton GE, Roweis ST (2007) Modeling human motion using binary latent variables. In: NIPS'06 Proceedings of the 19th international conference on neural information processing systems. pp 1345-1352

2. Safonova A, Hodgins JK, Polland NS (2004) Synthesizing physically realistic human motion in low-dimensional, behavior-specific spaces. ACM Trans Graph (TOG) 23:514-521

3. Yamane K, Nakamura Y (2008) Dynamics simulation of humanoid robots: forward dynamics, contact, and experiments. In: The 17th CISM-IFToMM symposium on robot design, dynamics, and control

4. Otten E (2003) Inverse and forward dynamics: models of multi-body systems. Philos Trans R Soc B 358:1492-1500

5. Motonaka K, Watanabe K, Maeyama S (2015) Kinodynamic notion planning for an X4-Flyer. In: Habib MK (ed) Handbook of research on advancements in robotics and mechatronics. IGI Global, Hershey, pp 455-474

6. Nolan L (2008) Carbon fiber prostheses and running in amputees: a review. Foot Ankle Surg 14:125-129

7. Grabowski AM, McGowan CP, McDermott WJ, Beale MT, Kram R, Herr HM (2010) Running-specific prostheses limit ground-force during sprinting. Biol Lett 6:201-204

8. Brüggemann GP, Arampatzis A, Emrich F, Potthast W (2009) Biomechanics of double transtibial amputee sprinting using dedicated sprinting prostheses. Sports Technol 1:220-227
9. Dumas R, Cheze L, Frossard L (2009) Loading applied on prosthetic knee of transfemoral amputee: comparison of inverse dynamics and direct measurements. Gait Posture 30:560-562

10. Rigney SM, Simmons A, Kark L (2015) Concurrent multibody and finite element analysis of the lower-limb during amputee running. IEEE EMBS Annu Int Conf 2015:2434-2437

11. Murai A, Endo Y, Tada M (2016) Anatomographic volumetric skin-musculoskeletal model and its kinematic deformation with surface-based SSD. IEEE Robot Autom Lett 1:1-7

12. Delp SL, Anderson FC, Arnold AS, Loan P, Habib A, John CT, Guendelman E, Thelen DG (2007) OpenSim: open-source software to create and analyze dynamic simulations of movement. IEEE Trans Biomed Eng 54:1940-1950

13. Nakamura Y, Yamane K, Fujita Y, Suzuki I (2005) Somatosensory computation for man-machine interface from motion capture data and musculoskeletal human model. IEEE Trans Robot 21:58-66

14. Rasmussen J, Damsgaard M, Surma E, Christensen S, de Zee M, Vondrak V (2003) AnyBody - a software system for ergonomic optimization. In: Fifth world congress on structural and multidisciplinary optimization

15. Blickhan R (1989) The spring-mass model for running and hopping. J Biomech 22:1217-1227

16. DerrickTR, Caldwell GE, Hamill J (2000) Modeling the stiffness characteristics of the human body while running with various stride lengths. J Appl Biomech 16:36-51

17. Endo Y, Tada M, Mochimaru M (2014) Dhaiba: development of virtual ergonomic assessment system with human models. Digit Hum Model 2014:1-8

18. Funken J, Willwacher S, Böcker J, Müller R, Heinrich K, Potthast W (2014) Blade kinetics of a unilateral prosthetic athlete in curve sprinting. In: 32 International conference of biomechanics in sports

\section{Submit your manuscript to a SpringerOpen ${ }^{\odot}$ journal and benefit from:}

- Convenient online submission

- Rigorous peer review

- Open access: articles freely available online

- High visibility within the field

- Retaining the copyright to your article

Submit your next manuscript at $\boldsymbol{\nabla}$ springeropen.com 